\title{
Une approche socio-pragmatique du dys/fonctionnement du rituel des salutations en français
}

\author{
Ruth De Oliveira \\ University of Cape Town
}

\begin{abstract}
Résumé. Ce travail porte sur le rituel des salutations dans les interactions sociales quotidiennes en contexte francophone. Sur la base d'un corpus de données authentiques - appréhendées selon une approche théorique combinée - nous nous proposons de démontrer dans quelle mesure la formule « bonjour » est une expression formelle et codifiée. Sa fonction étant de régler tout contact social, «bonjour » joue le rôle de garant de solidarité de l'individu et / ou d'appartenance de l'individu à son ou à cet environnement social de référence. En ce sens, «bonjour» est un performatif utilisé pour accomplir un acte institutionnel (i.e. saluer) lié à une convention humaine (i.e. Salutations). Parce que «bonjour» est le produit d'utilisation strictement dialogique, une fois cette formule adressée, une réponse sous forme d'une formule considérée comme une réponse équivalente est requise. En cas de dysfonctionnement, que se passe-t-il en termes de relations interpersonnelles / interculturelles ? Quelques résultats préliminaires suggèrent que dans certaines situations de communication, lorsque la symétrie de ce rituel est rompue et l'acte échoue, les locuteurs francophones métropolitains attribuent à [ce qu'ils entendent comme] la politesse une note plus élevée qu'à la coopération donnant lieu au phénomène inverse, c'est-à-dire, l'impolitesse.
\end{abstract}

\begin{abstract}
A socio-pragmatic approach to the ritual of greetings in French. This work deals with the ritual of greetings in daily social interactions in the French-speaking context. On the basis of a corpus of authentic data - apprehended according to a combined theoretical approach - we propose to demonstrate to what extent the formula "bonjour" is a formal and codified expression. Its function being to regulate all social contact, "bonjour" plays the role of guarantor of solidarity of the individual and/or of belonging of the individual to his/her or this social environment of reference. In this sense, "bonjour" is a performative used to perform an institutional act (i.e to greet) linked to a human convention (i.e. greetings). Because "bonjour" is the product of a strictly dialogical use, once this formula is addressed, a response in the form of a formula considered as an equivalent response is required. In the event of a dysfunction, what happens in terms of interpersonal / intercultural relations ? Some preliminary results suggest that in certain communication situations, when the symmetry of this ritual is broken and the act fails, French-speakers attribute to [what they understand as] politeness a higher rating than to cooperation giving rise to the opposite phenomenon, that is, impoliteness.
\end{abstract}




\section{INTRODUCTION}

Les interactions verbales sont des activités sociales qui trament nos relations quotidiennes et forgent ainsi nos cultures ${ }^{\mathrm{i}}$. Vu sous ce prisme, l'échange verbal est un fait social ${ }^{\text {ii }}$ régi par un système de normes établies pour et par nos sociétés ${ }^{\text {iii }}$.

Ces normes interactionnelles (ou compétences communicatives) - à la fois extérieures (savoirs socio-culturels) et subordonnées au langage (savoirs linguistiques) transitent par l'éducation dont le rôle institutionnel est de transformer l'humain en individu social ${ }^{\text {iv }}$.

Dans ce sens, les normes interactionnelles sont d'ordre moral puisqu'elles dictent aux locuteurs leurs façons de penser la communication en termes de conduite. Hormis des bouleversements sociaux profonds, un système de normes - réservoir de ressources communicatives mais aussi de contraintes - n'est pas modifiable par la seule volonté de l'individu ${ }^{\mathrm{v}}$.

Néanmoins, en admettant qu'il y ait dans cet - comme dans tout autre - ordre moral un désir de morale, on admet que pendant son parcours de socialisation l'individu s'y adapte parce qu'il croit bien agir ${ }^{\text {vi }}$. Comme le souligne Vion $(1996: 2)$ « les sujets ne sont pas les simples applicateurs d'un ordre préétabli mais contribuent, par leurs actions quotidiennes et leurs « conversations », à construire l'ordre social effectif. ». Le code se revêt ainsi en bien commun, à la fois obligatoire et objet de désir.

Parce qu'il est « impossible de ne pas communiquer » vii , l'individu socialisé interagit continuellement ${ }^{\text {vii }}$ pour accomplir des tâches spécifiques (par exemple, rechercher ou donner des informations, acheter ou vendre, transmettre ou interpréter des connaissances, prendre/donner des rendez-vous, trouver/offrir un emploi...) ou pas (par exemple, la conversation informelle). Aussi, chaque type [d'interaction] " commande " des fonctionnements langagiers et comportementaux relativement spécifiques (Vion 1996). Alors que l'hétérogénéité typologique des cadres interactifs possibles « [...] pourrait être allongée ad libitum ", (Kerbrat-Orecchioni et Traverso 2004 : 41), on y observe une constance (micro), le rituel des salutations. Autrement dit, en plus d'être un type d'interaction en soi (i.e. échange de salutations), ce rituel est à la base de toute interaction (i.e. salutations comme formules ouvrantes). Dans un cas comme dans l'autre, sa fonction sociale est, sinon d'assurer l'optimalité des relations interpersonnelles, du moins ne pas les détériorer. En ce sens, les salutations intègrent les codes de politesse : ceux qui ne s'y conforment pas - ne serait qu'en termes de coopération (Grice [1975]1979) - s'exposent à des sanctions et / ou génèrent des conflits sociaux de tout ordre. À l'instar de Leech (1983), on ne saurait en effet voir la politesse et la coopération comme des principes interactifs contradictoires alternant la priorité en fonction des exigences du contexte. Comme Leech le dit dans sa formule célèbre, " si vous n'êtes pas poli avec votre voisin, le canal de communication entre vous s'effondrera et vous ne pourrez plus emprunter sa tondeuse ». (1983:82)

Dans cette contribution, nous nous intéressons au rituel des salutations dans les interactions sociales quotidiennes en contexte francophone métropolitain. A l'appui d'un corpus de données authentiques appréhendées selon une approche théorique combinée (Cf. infra) nous nous proposons de démontrer dans quelle mesure la formule « bonjour » est une expression formelle et codifiée qui s'impose aux cadres communicationnels. Sa fonction étant de réguler tout contact social, « bonjour » joue le rôle de garant de la solidarité ix et/ou de l'appartenance de l'individu à « son » ou à « cet » environnement social de référence. En ce sens, dire « bonjour » est un performatif servant à accomplir un acte institutionnel (i.e. saluer) lié à une convention humaine (i.e. les salutations). Du fait que « bonjour » s'inscrit " dans un usage strictement dialogique » (Mejri 2017 :53) une fois cette formule adressée, une réponse " sous forme de formule considérée comme équivalente » est exigée (Mejri 2017 : 54). « Cette exigence d'équivalence » [A 
: Bonjour $\leftrightarrow$ B : Bonjour] est, comme le souligne Mejri (2017 : 54), « le pendant linguistique du respect mutuel véhiculé par l'acte de politesse. » En cas de dysfonctionnement [A. Bonjour $\leftrightarrow$ B : Ø/ A. Ø $\leftrightarrow$ B : ?], qu'en est-il des relations interpersonnelles? Que se passe-t-il au niveau interculturel?

Pour apporter des éléments de réponse à cette question, dans (2) nous passons en revue les notions fondatrices du cadre fonctionnel de nos propos, celui des études interactionnelles, et y faisons le point sur ses principaux apports à la pragmatique. Cela nous amène à retracer les moments clés de l'évolution du concept de politesse (2.1)

La troisième partie est consacrée au rituel des salutations, en particulier, au cas de l'échange de «bonjour » (3.1): ses caractéristiques et valeurs socio-culturelles (3.1.1).

Dans la dernière section, nous présentons (4.) et discutons (4.1) nos données selon la théorie des actes de langage. Quelques résultats préliminaires (4.2 et 4.3) suggèrent que dans certaines situations interlocutives lorsque la symétrie de ce type d'échange est brisée et que l'acte échoue, les participants métropolitains attribuent à [ce qu'ils entendent comme] la politesse une note plus élevée qu'à la coopération, suscitant le phénomène inverse, c'est- à-dire l'impolitesse.

\section{2 ÉTUDES INTERACTIONNELLES ${ }^{x}$ ET APPORTS PRAGMATIQUES}

Dans sa «mise en scène de la vie quotidienne » Goffman (1973) pose qu'interagir avec l'autre représente un double risque : celui de donner une image négative de soi et celui d'envoyer à l'autre une image négative de lui-même. Aussi, pour réussir l'enjeu interactif, il faut savoir ménager la face des autres sans perdre la sienne. Ce ménagement incessant et réciproque des faces en interaction est un processus de « figuration » (« face work »). Dans tous les cas, selon Goffman (1974), « alors même que la face sociale d'une personne est souvent son bien le plus précieux et son refuge le plus plaisant, ce n'est qu'un prêt que lui consent la société : si elle ne s'en montre pas digne, elle lui sera retirée ${ }^{x i}(1974: 13)$.

A partir des années 70, lorsque les linguistes commencent à s'intéresser aux interactions, Grice ([1975] 1979) pose que le but principal recherché dans les conversations est « une efficacité maximale de l'échange d'information » xii. Pour y parvenir, tout locuteur est tenu de respecter le contrat de coopération et ses maximes conversationnelles xiii. D'après le modèle gricéen, toutes sortes « d'autres règles (esthétiques, sociales ou morales) du genre : "soyez poli »» (1979:62) seraient dénouées de validité générale ${ }^{\text {xiv }}$. Autrement dit, le modèle gricéen ne prendrait pas en compte les paramètres interactifs socio-affectifs et appréhenderait la politesse comme un phénomène périphérique. Elle n'oriente[rait] pas l'efficacité du discours mais la gestion des relations ${ }^{\mathrm{xv}}$.

Les travaux de R. Lakoff sur la politesse (notamment 1977) s'inscrivent ainsi « en faux contre les maximes conversationnelles de Grice » (Manno 2002:8) dans le sens où l'auteure propose « des règles de compétence pragmatique » en guise $\mathrm{d}$ ' « ajustement » [du CP et ses maximes]. A savoir, i) soyez clair ${ }^{\text {xvi }}$ (centrée sur l'intention de communiquer sans ambiguïté), ii) soyez poli (centrée sur la relation entre participants de l'échange selon leur statut : plutôt que d'être clair, le locuteur met l'accent sur la 
politesse). En cas de conflit entre clarté et politesse, la politesse l'emporte car dans une conversation, il est plus important d'éviter l'offense que de transmettre le message avec clarté $^{\text {xvii }}$.

Cette reconnaissance de la centralité de l'aspect relationnel marque le dépassement de la conception traditionnelle purement informationnelle de la communication. Depuis, le champ d'études sur la politesse n'a de cesse d'évoluer.

\subsection{De la politesse à l'im/politesse en interaction}

Dans ses Principles of Pragmatics, Leech (1983) écrit que toute interaction est soumise au réquisit fondamental « soyez poli ». Bien que paru dans les années 80 , cet ouvrage intègre les principes pragmatiques annoncés quelques années auparavant dans son texte Language and tact (1977). Comme souligné par Terkourafi (2015)

« La correspondance entre le sens (sémantique) et la force (pragmatique), le caractère indirect et la politesse en tant que notion scalaire y sont tous discutés. Pour Leech, la politesse (ou plutôt le tact) concerne l'évitement stratégique de conflits et le respect des autres."

Il en ressort la caractéristique fondamentale de la politesse : son caractère asymétrique (i.e. ce qui est poli pour le locuteur peut être vu comme impoli pour l'allocutaire et vice versa). Leech envisage les comportements interactionnels selon une échelle de coûts et bénéfices : plus le bénéfice est en faveur du récepteur, moins impoli sera l'acte en question. Aussi, d'après ses maximes, pour donner une image valorisante de soi, l'objectif n'est pas l'être (mœurs réelles) mais paraitre (mœurs oratoires).

Depuis les années 80, la théorie linguistique de la politesse de Brown et Levinson (1987) sert de base fondamentale à la plupart des travaux axés sur les différents supports linguistiques de la politesse, notamment : actes de langage, constructions syntaxiques, éléments lexicaux, cherchant entre autres à les comparer culturellement, à tenter d'identifier des universaux (Meier 1995). D'après les concepts inhérents à ce modèle basés à la fois sur les principes du "travail de figuration » initié par Goffman en sociologie, sur ceux du CP élaborés par Grice en domaine logico-philosophique de même que sur les PP avancés par Leech - dans un cadre interactif donné, les manifestations de politesse (i) reflètent les relations sociales que les participants entretiennent entre eux ; (ii) servent à modifier ou à consolider des relations préexistantes. Influencée par deux facteurs, à savoir la proximité (dans le sens affectif du terme) et les relations hiérarchiques entre les participants ; (iii) la politesse comme d'ailleurs l'impolitesse peuvent (i) être négatives ou positives, (ii) viser la face positive (l'estime de soi) ou la face négative (le désir de liberté, le territoire) des interactants. ${ }^{\text {xiii }}$

La profusion des recherches autour de la politesse linguistique mène au constat de l'impact que ce concept exerce sur les opérations de production et interprétation des énoncés échangés. Et ce, comme le note Kerbrat-Orecchioni, "au même titre que les règles plus spécifiquement linguistiques, et que les maximes conversationnelles de Grice » (1992 : 160). De ce fait, il est juste d'affirmer que dans le domaine interactionnel, la politesse est un phénomène pertinent linguistiquement (et pas seulement). Par ailleurs, pour de nombreux chercheurs, « il est impossible de décrire efficacement ce qui se passe dans les échanges communicatifs sans tenir compte de certains principes de politesse » 
(Kerbrat-Orecchioni 1992 : 159).

A ce propos, si la base conceptuelle du domaine reste l'affaire de la pragmatique, d'autres modèles de politesse continuent d'émerger dans des travaux menés dans bien d'autres disciplines, notamment en psychologie sociale, en sociologie, dans les études de communication. ${ }^{\text {xix }}$ " À la lumière des preuves actuelles », Kasper écrit (1990) qu'

« il est également devenu évident que les premiers modèles, bien qu'impressionnants par leur parcimonie et leur élégance, sont trop simplistes. Leur réalisation durable est d'avoir fourni d'excellentes heuristiques pour enquêter sur un objet d'enquête très complexe. En tant que théories revendiquant l'universalité, elles doivent être élaborées et révisées. » $(1990: 1)^{\mathrm{xx}}$

Sur ce dernier point, au-delà de la critique sur sa prétention à l'universalitéexi , pour « rendre [le modèle classique] plus efficace », nombreux sont les remaniements proposés : dont ceux notoires par Kerbrat-Orecchioni (2010) :

« l'admission de la notion de Face Flattering Acts aux côtés de celle de Face Threatening Acts ; l'approfondissement de la réflexion sur les phénomènes d'impolitesse ; l'introduction dans le système de catégories venant s'ajouter à l'opposition binaire politesse/impolitesse, comme les catégories de l'« hyperpolitesse», de la « non-politesse » et de la « polirudesse ».

Ces propositions " d'améliorations », notamment en ce qui concerne le développement de la réflexion sur l'impolitesse, de nombreux apports récents permettent de constater non plus « un certain basculement » (Kerbrat-Orechionni 2010: 38) mais le basculement certain de l'intérêt de la politesse vers l'impolitesse. Sifianou (2010) écrit à cet égard que « l'année 2008 pourrait être appelée « l'année de l'impolitesse » (2010:119). À l'auteure de présenter les arguments suivants :

« La parution d'un livre (Bousfield, Impoliteness in Interaction), [d'un] volume édité (Bousfield \& Locher (ed), Impoliteness in language: Studies on its interplay with power in theory and practice], de deux numéros de revues consacrés [à la politesse] (Journal of Politeness Research 4.2 et Pragmatics 18.4) ne peut être une simple coïncidence. Une telle prolifération est la récolte exceptionnelle des plaidoiries de nombreux chercheurs pour lesquels l'étude de l'impolitesse mérite l'attention. » $(2010: 119)^{x x i i}$

Aussi, de 2002 à 2018, 21 conférences ont été consacrées à ce concept. Depuis 2009, l'impolitesse bénéficie également des travaux en ligne de Jonathan Culpeper (http://www.lancs.ac.uk/fass/projects/impoliteness/) ${ }^{\text {xxiii }}$. Sous le titre Impoliteness: Using and Understanding the Language of Offence, Culpeper se propose de traiter les faits de langue généralement considérés comme antisocial, et étiquetés à l'aide d'un vaste éventail de termes tels que impoli, grossier, inconsidéré, injurieux, etc. ${ }^{\text {xxiv }}$

A partir de questionnements théoriques et tentatives de conceptualisations de ce concept, on a vu émerger une autre dimension de l'im/politesse dans les interactions verbales, à savoir le constituant émotionnel. ${ }^{\mathrm{xxv}}$

De ce qui précède, on retient tout d'abord que l'interaction verbale n'est pas un processus mécanique de la parole mais une activité sémantique, un processus de création de sens, d'échange de significations motivé par des raisons fonctionnelles et pas seulement. Aussi, à l'exception de quelques situations particulières ${ }^{x x v i}$, la politesse 
s'affirme comme le ciment de tout échange, et ce malgré la quantité troublante de divergence et/ou de flou sur le sens du concept lui-même xxvii comme sur le sens des concepts concomitants, dont celui d'impolitesse.

Enfin, bien qu'il soit devenu indiscutable que les maximes de Grice, autrement dit, les éléments fondateurs du principe de coopération en interaction, ne s'appliquent pas de manière identique dans toutes les sociétés ${ }^{\text {xxviii }}$, des spécialistes (dont Meier 1995) nous rappellent que ce que toutes les sociétés ont en commun, c'est l'existence de normes pour un comportement approprié. Identifier ces normes et leurs motivations sous-jacentes reste l'affaire de la pragmatique dont le développement des travaux, y compris au niveau des relations interpersonnelles comme interculturelles, contribuent à une meilleure connaissance de l'humain dans les sociétés humaines.

Dans (3) l'échange de «bonjour» est abordé en tant qu'acte performatif. Bien que sa réalisation ne requière ni spontanéité ni sincérité son paramétrage symétrique présente l'interaction, à l'instar de ce qu'on appelle en linguistique interactionnelle action et réaction, comme un réseau d'influences mutuelles ${ }^{\text {xxix }}$. Compte tenu de son haut degré de ritualisation, nous posons que le dysfonctionnement de celle-ci constitue une menace pour l'identité collective à l'intérieur comme à l'extérieur de cette collectivité.

\section{RITUEL DES SALUTATIONS ROUTINIERES}

Le terme « rituel » est issu du latin ritus qui signifie « ordre prescrit ». Les différents rituels sociaux prennent naissance, selon Jacques Cortès (2007 :154)

« [...] dans les grandes influences historiques dont un peuple a été imprégné et conditionné culturellement dès l'enfance. La ritualisation s'est installée progressivement et les postures et mots qui l'accompagnent sont devenus la Norme. Des variations de surface nombreuses, des évolutions, des révolutions, des rejets conjoncturels, des inventions...peuvent affecter cette norme mais rien, je crois, ne remet en cause ses principes fondateurs. »

Dans Politesse, savoir-vivre et relations sociales (2014), Dominique Picard établit le lien entre politesse et rituel, que l'auteure définit ainsi :

« [rituels :] actes répétitifs et quasi-automatiques, ne pouvant jamais se réduire à de simples actes opératoires, ayant une forte charge symbolique et s'appuyant sur une adhésion à des valeurs sociales et culturelles, [...] les rituels participent pleinement au système institutionnel, dont ils sont issus. » (Picard 2014 : 48).

D'après cette définition, les caractéristiques fondamentales du rituel de politesse sont : sa dimension mécanique, son caractère symbolique et son aspect institutionnel.

\subsection{Le cas de « bonjour »}

A l'égard de sa dimension mécanique, l'échange de «bonjour » est un acte répétitif et quasi-automatique appris ou mimé, en tout cas, intériorisé très tôt par les locuteurs francophones tant en milieu familial que scolaire. Cette dimension mécanique, «c'est ce qui garantit au système des relations sociales une prédictibilité économisant à la société bien des conflits. » (Mejri 2017 : 20). En ce sens, une fois réalisé avec succès, l'échange 
de « bonjour » est à même de « transformer les conflits en symboles en les ordonnant et en les intégrant dans des scénarios préétablis » (Mejri 2017 : 20). Aussi, au niveau symbolique, le respect de ces scénarios garantit, selon la définition proposée de Mejri « des relations présentant le minimum de conflit et le maximum de respect entre les uns et les autres, respect inscrit dans les valeurs que le système érige en rituels. » (Mejri 2017 : 20). Ces caractéristiques mécanique et symbolique sont à notre avis indissociables de sa fonction institutionnelle dont le rôle est de faire accepter aux utilisateurs de la langue l'ensemble des règles ritualisées.

\subsubsection{Repères socio-culturels}

En guise d'illustration, soit le cas de figure (ou scénario) où l'on voit un enfant se faire réprimander parce qu'il n'a pas salué une personne ${ }^{\mathrm{xxx}}$. Lorsque formulée par les parents, la réprimande se manifeste généralement par l'injonction « dis bonjour à la dame/ au monsieur »; quand énoncé par celui/celle « ignoré(e) » par enfant, le blâme (dont le ton plaisantin ne saurait masquer le reproche) se manifeste par (entre autres) «Alors, on a perdu sa langue / ne dit plus bonjour à mamie ? ». Dans un cas comme dans l'autre, ce qu'on veut clairement faire comprendre à l'enfant est qu'il a commis une faute et que pour que cette faute soit réparée il faut qu'il prononce le mot «bonjour ». Si l'enfant maintient sa position (entre autres, parce qu'il est fatigué ou distrait) et refuse d'accomplir le rituel ${ }^{\mathrm{xxxi}}$, on lui dit qu'il n'est pas gentil, qu'il n'est pas poli ; ou alors on affirme qu'il est poli / gentil [par conséquent] il dit « bonjour » à la dame / au monsieur. Un survol du parcours étymologique du qualificatif « gentil» nous rappelle son origine « noble », " noble par son comportement»; tandis que poli renvoie à « civilisé ». La circularité sémantique qu'entretiennent ces qualificatifs (gentil.le, noble, poli.e, civilisé.e) fait ressortir le profil valorisant de celui/celle qui se plie au rituel du «bonjour».

Il est ainsi tout à fait défendable la thèse selon laquelle, arrivés à l'âge adulte, ces locuteurs francophones s'appliquent à leur tour à la transmission dudit rituel, malgré les contraintes subies pendant leurs parcours de socialisation ${ }^{\text {xxxii }}$. Et ce, parce qu'ils ont appris à valoriser l'acte de saluer, non pas ou non plus, comme une règle comportementale imposée mais comme un savoir-vivre, à la française ${ }^{\text {xxxiii. }}$. Si (ou compte tenu du fait que) dire «bonjour" c'est la moindre des politesses, on fait l'hypothèse que lors des interactions quotidiennes, en vue du maintien de ce (que nous appellerions) culte de la ritualisation de l'échange de «bonjour», le locuteur francophone joue le rôle de «porte-parole autorisé » [...] sa parole [concentrant] le capital symbolique accumulé par le groupe qui l'a mandaté et dont il est le fondé de pouvoir » (Bourdieu $1982: 109)$.

Des trois caractéristiques abordées (supra), il en ressort au moins trois fonctions essentielles de l'échange rituel de «bonjour » : il s'agit de marquer le respect à travers une recherche constante d'équilibre en fonction de la nature des relations, ce qui, pour le coup, permet de préserver les liens sociaux (Cf. Picard 2014). Dire «bonjour» consiste ainsi à faire quelque chose ; selon la terminologie austinienne, dire «bonjour » est une énonciation performative ou encore, c'est un «performatif ».

\section{LE RITUEL DU « BONJOUR » : UN ACTE DE LANGAGE}

Dans le cadre de la théorie des actes de langage, en énonçant «bonjour » on accomplit, en fait, trois actes simultanés : 
- un acte locutoire, i.e. on articule et combine des sons, on évoque et relie les notions représentées par le terme « bonjour»;

- un acte illocutoire, i.e. en énonçant « bonjour » on transforme les rapports entre les interlocuteurs : on accomplit l'acte de salutation, donc de politesse ;

- et un acte perlocutoire, i.e. cette énonciation vise des effets plus lointains : en énonçant « bonjour » on attend une réponse.

Dans cette perspective, l'échange de «bonjour » intègre un type d'action " qui affecte, altère, ou maintient les relations de soi et d'autrui dans la communication de face à face » (Labov et Fanshel 1977 : 59). Aussi, « bonjour » semble bien appartenir à la classe de ce que Kerbrat-Orecchioni (1995 : 72) appelle les relationèmes, c'est-à-dire des éléments « à considérer à la fois comme des indicateurs, et des constructeurs de la relation interpersonnelle. »

De son côté, Andersson (1975) suggère trois manières - plus ou moins différentes selon lesquelles une action peut être identifiée comme un acte illocutoire. Dans ce qui suit nous nous appuyons sur Andersson pour démontrer que dire « bonjour » est un acte " illocutoire » du fait d'être une action (i) " communicative ", (ii) " performante socialement » ou (iii) « cérémonielle » $(1975: 112)$.

\subsection{Analyse des données}

\subsubsection{Le corpus}

Le point de départ de notre étude sont les données suivantes :

« Bonjour, un café, s'il vous plaît »:1 $€$.

«Un café, s'il vous plaît » : 1,30€.

«Un café » : $1,50 €$.

Ces données proviennent d'une ardoise de prix du café proposés par un restaurateur grenoblois, au nom d'une « politique de prix calculée selon l'indice d'amabilité » des clients (Le Parisien 13/03/2016 ${ }^{\text {xxxiv }}$ ). Prise en photos par des clients ${ }^{\text {xxxv }}$ et postée sur diverses réseaux sociaux l'ardoise a fait le tour de la toile déclenchant environ 4000 commentaires d'internautes Français, francophones et étrangers ${ }^{\text {xxxvi }}$. Dans une démarche analytique qualitative, notre approche du corpus initial (ci-haut) tient compte des premières 400 réactions des internautes, à savoir environ $10 \%$ des données rassemblées, archivées et disponibles sur la plateforme Reddit ${ }^{\text {xxxvii }}$.

\subsection{Quelques constats argumentés}

À première vue, les informations exposées sur l'ardoise semblent servir de rappel aux clients (natifs ou non) qui dans cette situation interactive précise, pour une raison quelconque, n'emploient pas les séquences prescrites et construites conformément à des règles de procédure strictes et rigoureuses (Cf. Kerbrat-Orecchioni 2005). Ces expressions dites de signification procédurale (ESP) telles les salutations (ici, «bonjour») sont en effet considérées comme partie intégrante (autrement dit, nécessaires) dans les requêtes et / ou les commandes (à l'exemple de corpus de départ proposé plus haut). Le contenu des ESP passe au deuxième plan, seul leur rôle fonctionnel compte. En français, beaucoup de ces expressions / mots ont été pragmaticalisés et sont appris (Cf. repères socio-culturels) par les autochtones grâce à un 
processus de socialisation dans leur « usage correct». Pour soutenir ces constats, voici quelques témoignages :

a. «Les habitués nous saluent, donc on en tient compte. Mais certains touristes qui arrivent en été ne nous respectent pas, et quand on entend 250 fois café ! ça nous rend grognons. Il ne faut pas qu'ils s'étonnent après d'avoir une addition un peu salée ». [Avis d'un restaurateur ${ }^{\text {xxxviii }]}$

b. «Rien n'empêche [...] l'amabilité d'être un critère » [...] C'est un clin d'œil parmi d'autres pour rappeler que les cafés sont des lieux de sociabilité. Et ils en ont bien besoin, eux qui sont passés de 200000 à moins de 35000 en cinquante ans. " [Roland Héguy, président de l'Union des métiers et des industries de l'hôtellerie xxxix ]

c. « C'est un système que nous ferions bien d'adopter ailleurs que dans les restaurants. Si des individus impolis dérogent aux formules conventionnelles d'interaction humaine, nous devrions le leur faire savoir. $»{ }^{\mathrm{xl}}$

Ces témoignages ci-haut permettent d'identifier le rituel du «bonjour » comme un acte illocutoire. A l'appui des travaux de Andersson (1975) nous posons que ces témoignages ( $\mathrm{a}, \mathrm{b}$ et $\mathrm{c}$ ) démontrent que dire «bonjour » est une action "communicative" parce qu'elle est acceptée dans une certaine communauté (ici, en France métropolitaine) comme étant censée fournir « une preuve solide » que la personne qui commet l'acte a une conviction, un désir ou un sentiment similaire, et qu'elle a l'intention de faire savoir au public qu'elle a cette conviction, ce désir ou ce sentiment similaire, et qu'elle veut que le public sache qu'elle a cette conviction, ce désir ou quoi que ce soit de similaire (e.g. a et b). Aussi, dire «bonjour » est une action " performante socialement » et " cérémoniale » parce qu'elle est acceptée dans cette communauté donnée comme une preuve solide que la personne qui commet l'acte a l'intention de créer et provoque effectivement un certain état normatif, ou ce qui est dans l'intérêt de cette communauté (e.g. c).

D'autre part, les non-natifs (surtout ceux n'ayant pas passés par le même processus de socialisation) peuvent ne pas connaitre ou ne pas faire usage du même éventail d'ESP que les francophones. Étant donné que chaque langue / communauté a ses propres règles pragmatiques et contextes dans lesquels elles sont appliquées, les informations exposées sur ces ardoises, à savoir le prix du café, apparaissent comme une attaque car elles avertissent clairement les clients des sanctions imposées en cas de non-respect des règles en vigueur. En voici quelques témoignages :

i. «Je me demande ce qui se passerait [...] si vous disiez « bonjour, désolé de vous déranger, mais je me demande s'il vous serait possible de m'apporter un café, je vous en serais éternellement reconnaissant... ». Vous pay[eriez] ? » ${ }^{x l i}$

ii. "I have French coworkers I have to deal with. When they piss me off, I will go about a week without any greetings. Its my passive aggressive loathing of them. They are actually really cool, but I laugh out loud when I dont send a greeting. " xlii

iii. "I don't get it. Why is there variance in the price?» xliii

iv. "Haha, manners..." xliv 


\author{
v. "Well isn't that nice!"” xlv \\ vi. "I'd order the expensive one every time. Fuck their patronizing attitude." xlvi
}

Par le choix des mots, ces diverses réactions témoignent de différents positionnements visà-vis du code établi, à savoir l'ironie (une ironie mêlée de critique sociale à peine voilée, e.g. i et v), la moquerie (ii et iv), l'étonnement (iii) et l'agacement (vi), ce positionnementci fait par ailleurs ressortir l'attitude défiante des internautes à l'égard des "menaces » pressenties.

\title{
4.3 Considérations finales préliminaires
}

Sous le terme générique de livres de courtoise (en anglais manners), les préceptes de politesse ont d'abord servi à instruire les enfants princiers. Élaborés par des érudits (Cf. Erasmus 1530, La Bruyère 1688) ils ont ensuite été adressés aux fils des classes supérieures, avant d'influencer tous les enfants et d'être censés être suffisamment universels pour servir les sociétés elles-mêmes. (Cf. Picard 2014).

De la Renaissance à la Révolution française, les mœurs (ou manners/manières) ont été un aspect essentiel de l'idéal de la civilisation. Ces ouvrages dits de courtoisie étaient les vecteurs littéraires des discussions sur des sujets/concepts diversifiés tels la morale, la défense des idéaux de caractère, de caractéristiques, d'habitudes, de manières, d'accomplissements... en somme de tout ce qui entoure «l'art de vivre en société ».

Au-delà de son caractère prescriptif, l'échange de salutations, dont le rituel du « bonjour », fait ainsi écho à un système de valeurs traditionnelles valorisé et valorisant.

L'analyse qualitative des données de notre corpus suggère qu'en France (Cf. réactions [surtout] en français) le bonjour de politesse reste une valeur contemporaine sûre que l'on s'efforce de défendre quelles qu'en soient les conséquences, y compris être considéré comme impoli (Cf. réactions en langues française et [surtout] anglais). 


\section{Références bibliographiques}

Austin, J.L. ([1962] 1970). Quand dire c'est faire. Paris : Éd. du Seuil.

Boujou, M. (2011). Petites chroniques à mots couverts. Paris :Bouquineo.

Burke, P. (1999). Les langages de la politesse. Terrain, $33 \mid$ septembre, p.111-126.

URL : http://terrain.revues.org/2704

Brown, P., Levinson, S. C. (1987). Politeness: Some universals in language usage. Cambridge : Cambridge University Press.

Culpeper, J. (2013). Impoliteness: questions and answers. Aspects of linguistic impoliteness, 2-15. Denis Jamet et Manuel Jobert (eds.). Cambridge : Cambridge Scholars Publishing.

Culpeper, J. (2011). Impoliteness : Using language to cause offence. Cambridge : Cambridge University Press.

Durkheim, E. (1967 [1894]). Les règles de la méthode sociologique. Version numérique. Québec : Les classiques des sciences sociales. URL :

http://classiques.uqac.ca/classiques/Durkheim_emile/regles_methode/durkheim_regles_methode. pdf

Durkheim, E. (1974 [1925]). L'Éducation morale. Paris : PUF

Durkheim, E. (2001 [1917]). Introduction à la morale. Version numérique. Québec : Les classiques des sciences sociales.

$\mathrm{URL}:$ http://classiques.uqac.ca/classiques/Durkheim_emile/textes_2/textes_2_10/durkheim_intro morale.pdf

Erasme (de Rotterdam). (1530). De civilitate morum puerilium. ("On civility in children"). URL : https://data.bnf.fr/en/15097638/erasme_de_civilitate_morum_puerilium/

Goffman, E. (1974). La mise en scène de la vie quotidienne. 1. La présentation de soi. 2. Les relations en public. Paris : Les Éditions de minuit.

Goffman, E. (1973). Les rites d'interaction. Paris : Les Éditions de Minuit.

Grice, P. ([1975] 1979). Logique et Conversation. Communications, 30. La conversation. pp. 57-62.

Kasper G. (1990). Linguistic Politeness: Current Research Issues. University of Hawai'i Working Papers in ESL, Vol. 9, No. 1, May. pp. 1-33.

Kerbrat-Orecchioni C. (2010). L'impolitesse en interaction. Lexis [En ligne], HS 2. URL: http://lexis.revues.org/796.

Kerbrat-Orecchioni, C., Traverso, V. (2004). Types d'interactions et genres de l'oral. Langages, vol. 153, no. 1. pp. 41-51.

Kerbrat-Orecchioni, C. (1998). La notion d'interaction en linguistique : origines, apports, bilan. Langue française, $n^{\circ} 117$. pp. 51-67.

Kerbrat-Orecchioni, C. (1995). La construction de la relation interpersonnelle : quelques remarques sur cette dimension du dialogue. Cahiers de Linguistique Française, 16, vol.1. pp. 69-88.

Kienpointner, M. (1997). Variety of rudeness. Types and functions of impolite utterances. Functions of Language, Vol. 4.2. pp. 251-287.

Labov W., Fanshel D. (1977). Therapeutic Discourse. New York : Academy Press.

Laberge, Y. (2016). La théorie d'Erving Goffman sur l'interaction face-à-face pour comprendre le téléphone mobile et les SMS. Recherches sociologiques et anthropologiques, 47-1. pp. 205-211.| URL : http://journals.openedition.org/rsa/1676

La Bruyère, J. de (1688). Les Caractères. Version numérique. URL: https://gallica.bnf.fr/essentiels/la-bruyere

Lakoff, R. T. (1977). What You Can With Words : Politeness, Pragmatics, and Performatives. Proceedings of the Texas Conference on Performatives, Presupposition, and Implicatures. A. Rogers et al (éds). Arlington : Center for Applied Linguistics. pp. 79-105.

Leech, G. (2004). Politeness: Is there an East-West Divide? Journal of Foreign Languages, n. 6, November. General Serial No.160. pp. 167-206. 
Leech, G. (1983). Principles of pragmatics. London : Longman

Manno, G. (2002). La politesse et l'indirection : un essai de synthèse. Langage et société, 2 ( $\left.n^{\circ} 100\right)$. pp. 5-47. URL : https://www.cairn.info/revue-langage-et-societe-2002-2-page-5.htm

Maulini, O. (2009). Mouche ton nez, dis bonjour à la dame ! Réflexions didactologiques sur la politesse et sur sa vision spéculaire, l’hypocrisie. Synergies Monde ${ }^{\circ}{ }^{\circ}$ 3. pp. 147-159

Meier, A.J. (1995). Defining politeness: universality in appropriateness. Language Sciences, Vol. 17, No. 4. pp. 345-356. URL : https://www.sciencedirect.com/science/article/pii/0388000195000194

Mejri, S. (2017). Les formules de politesse et de présentation. Paris : Garnier.

Picard, D. (2014). Politesse, savoir-vivre et relations sociales. Paris : Presses Universitaires de France.

Sifianou, M. (2010). Review of Impoliteness in language: Studies on its interplay with power in theory and practice. Derek Bousfield \& Miriam A. Locher (eds.). Language in Society 39. Berlin : Mouton de Gruyter.

Simon, A. C. (2000). Politesse et idéologie : rencontres de pragmatique et de rhétorique conversationnelles. Leuven : Peeters Publishers.

Terkourafi M. (2015). The pragmatics of politeness. By Geoffrey Leech. Language, volume 91, number $4 . \quad$ pp. 957-960.

URL : https://www.linguisticsociety.org/sites/default/files/09_91.4Terkourafi.pdf

Traverso, V. (2012). Analyses interactionnelles : repères, questions saillantes et évolution. Langue française, $3\left(n^{\circ} 175\right)$. pp. 3-17.

Vion, R. (1996). L'analyse des interactions verbales. Les Carnets du Cediscor 4. pp. 19-32.

${ }^{\mathrm{i}} \mathrm{La}$ « culture » est ici entendue dans une acception sociologique synthétique et générale du terme, à savoir " ce qui est commun à un groupe d'individus », " ce qui soude ", " ce qui est appris, transmis ».

11 Terme emprunté aux travaux d'Émile Durkheim (1967 [1894] : 44-45), fondateur du concept de fait social qu'il présente comme " toute manière de faire, fixée ou non, susceptible d'exercer sur l'individu une contrainte extérieure ; ou bien encore, qui est générale dans l'étendue d'une société donnée tout en ayant une existence propre, indépendante de ses manifestations individuelles. »

${ }^{111}$ Une « société » est « avant tout, un ensemble d'idées et de sentiments, de certaines manières de voir et de sentir, une physionomie intellectuelle et morale qui est distinctive du groupe tout entier. La société est, avant tout, une conscience : c'est la conscience de la collectivité » Durkheim 1974 [1925] : 236.

iv A ce propos, voici l'observation faite par Durkheim (1967 [1894] : 39) : « Quand on regarde les faits tels qu'ils sont et tel qu'ils ont toujours été, il saute aux yeux que toute éducation consiste dans un effort continu pour imposer à l'enfant des manières de voir, de sentir et d'agir auxquelles il ne serait pas spontanément arrivé. [...] c'est que l'éducation a justement pour objet de faire l'être social. »

V Selon Durkheim (2001 [1917] : 8) «L'idéal moral n'est pas immuable ; il vit, évolue, se transforme sans cesse, en dépit du respect dont il est entouré. » Il y a dans cette théorie de morale l'idée de libre arbitre et l'autonomie individuelle.

vi Dans le chapitre "Perdre la face ou faire bonne figure ?" Goffman écrit (1974:13) : «Par les attributs que lui sont accordés et la face qu'ils lui font porter, tout homme devient son propre geôlier. C'est là une contrainte sociale fondamentale, même s'il est vrai que chacun peut aimer sa cellule. »

vii « Il est impossible de ne pas communiquer ». Il n’y a pas de « non-comportement» (le silence et l'inaction sont un comportement). Par conséquent, la communication est 
permanente. Tel est le postulat de départ de l'École de Palo Alto dont les travaux portent sur une théorie de la communication et de la relation entre les individus.

vi1i Dans La notion d'interaction en linguistique (1998: 51) Kerbrat-Orecchioni débute son approche en citant J. Gumperz : " parler c'est interagir », et M. Baktine " l'interaction verbale est la réalité fondamentale du langage ».

1X Durkheim (notamment 1967 [1894]) a montré que la solidarité pouvait prendre des formes différentes, dont la solidarité mécanique, fondée sur la similarité des individus dans les sociétés traditionnelles à forte conscience collective.

${ }^{\mathrm{X}}$ Études interactionnelles, entre autres, Traverso (2012).

${ }^{x i}$ Quelques semaines avant sa mort, on découvre une sorte de testament académique sous le titre "L'ordre de l'interaction" rédigé en 1982 dans lequel Goffman reformule sa définition de l'interaction sociale : "L'interaction sociale peut être définie, de façon étroite, comme ce qui apparaît uniquement dans des situations sociales, c'est-à-dire des environnements dans lesquels deux individus, ou plus, sont physiquement en présence de la réponse de l'un et de l'autre (1982 : 230)." Cité par Laberge (2016 : 208).

Xi1 Dans ce sens, communiquer c'est transmettre un message contextualisé qu'un émetteur envoie à un récepteur, moyennant le choix d'un canal et l'adoption d'un code partagé.

Xi1i Trois portent sur le contenu (cf. quantité, qualité et relation), et une sur l'intelligibilité du discours (maxime de modalité ).

xiv Voici ce qu'en dit Grice (1979: 62) «Il y a aussi bien sûr toutes sortes d'autres règles (esthétiques, sociales ou morales), du genre «Soyez poli », que les participants observent normalement dans les échanges parlés, et qui peuvent donner lieu à des implicitations non conventionnelles. Toutefois, les règles conversationnelles, et les implicitations qui leur sont attachées, sont plus spécialement accordées, je le pense, aux buts particuliers pour lesquels la parole (et donc les échanges de parole) est faite et d'abord employée. J'ai énoncé ces règles en supposant que le but recherché soit une efficacité maximale de l'échange d'informations ; cette définition est bien sûr trop étroite, et il faudrait généraliser ce schéma de règles de façon à pouvoir tenir compte de buts aussi généraux que le désir d'influencer ou de mener les autres. »

$\mathrm{XV}$ À ce propos, Simon (2000).

${ }^{\mathrm{x} v i}$ La maxime «soyez clair » est inspirée du CP de Grice. En ajoutant sa deuxième règle « soyez poli » Lakoff suggère les « règles de la politesse » suivantes : 1) Ne vous imposez pas ; 2) Donnez le choix ; 3) Mettez votre interlocuteur à l'aise.

xvii Dans les mots de Lakoff $(1977: 86)$ : "We have seen [...] that recourse to syntactic explanations won't help us understand much of language use; we must, in fact, look at the pragmatic aspect of communication and ask what communicative effect is achieved by the use of these devices. We find that there are, indeed, two basic motivating forces that cause us to adhere to or violate our syntactic rules of pragmatic competence are : (1) Make yourself clear. - (2) Be polite. These are the pillars of our linguistics as well as non-linguistic interactions with one another."

xviii Soulignons avec Meier que "Brown and Levinson [...] themselves, in their introduction, prove to be the strongest critics of their own concepts: [...] 'our strategies were never intended as an exhaustive taxonomy of utterance styles [...] and they therefore do not necessarily provide sensible categories for quantitative esearch'. The authors then proceed to point out that politeness 'markers' are not quantifiable signals of politeness and that the use of politeness strategies may have a motivation other than politeness. What emerges in the 1987 introduction then is a multifunctionality, a relativity, a dependency on context that makes B/L's politeness model of little more value to empirical investigation than more general concepts of politeness, but which, due to the attractiveness of misleadingly clear, delineated categories of politeness strategies, has led researchers astray in their attempts to apply the model.” Meier 1995 : 351 
xix D'après Culpeper (2013 : 3) “[t]here are [...] important spin-offs for other disciplines, including social psychology (especially related to verbal agression), sociology (especially related to verbal abuse), conflict studies (e.g. resolution of verbal conflict) and media studies (e.g. exploitative TV)."

$\mathrm{XX}$ " [...] in the light of current evidence, it has also become clear that the early models, while impressive in their parsimony and elegance, are over-simplistic. Their lasting achievement is to have provided excellent heuristics to investigate a highly complex object of inquiry. As theories with claims to universality, they require elaboration and revision." Kasper $1990: 1$.

xxi Dans Politeness: Is there an East-West Divide? (2004) Leech écrit : "The theory of politeness of Brown and Levinson $(1978,1987)$ has remained the most seminal and influential starting point for cross-cultural and cross-linguistic contrastive pragmatics. Yet it has also provoked countervailing arguments from Ide (1989, 1993), Matsumoto (1989), Gu (1990), Mao (1994) and others, pointing out a Western bias in Brown and Levinson's theory, particularly in their construal of the concept of 'face', in their overemphasis on face-threat and their assumption of individualistic and egalitarian motivations, as opposed to the more group-centred hierarchy-based ethos of Eastern societies."

Xxi1 Dans les mots de Sifianou (2010 : 119): "The year 2008 could be referred to as "the Year of Impoliteness." The appearance of a book [Derek Bousfield, Impoliteness in Interaction. 2008] and [an] edited volume [Derek Bousfi \& Miriam A. Loch er (eds.), Impoliteness in language: Studies on its interplay with power in theory and practice], and two special journal issues (Journal of Politeness Research 4.2 and Pragmatics 18.4) cannot be a mere coincidence. Such proliferation is the bumper harvest of pleas by many scholars that the study of impoliteness deserves attention."

XX111 En fait, ces recherches en ligne sont un condensé de Culpeper, J. (2011).

xxiv Culpeper précise que ses travaux en ligne "[...] focuses on language that is generally considered anti-social, and is labelled by a wide-range of terms such as impolite, rude, inconsiderate, verbally abusive, etc. It aims at describing that language and explaining how it works." https://www.lancaster.ac.uk/fass/projects/impoliteness/index.htm

${ }^{\mathrm{XXV}}$ Entre autres, Wierzbicka (1999) sur la conceptualisation des émotions à travers les langues et les cultures, Blum-Kulka et al $(1984,1989)$ sur la notion d'implication; Moise (2019) sur «Espace public et fonction de l'insulte dans la violence verbale».

Xxv1 Des situations où les actes impolis peuvent servir à resserrer les liens sociaux. C'est le cas, par exemple, des insultes rituelles entre jeunes de différentes cultures. Sur ce point, voir notamment Kienpointner (1997).

Xxvi1 Meier écrit à cet égard (1995 : 345) : “A closer look, [...], at both Brown and Levinson's [...] model and the subsequent literature reveals a disturbing amount of divergence and lack of clarity concerning the meaning of politeness and concomitant concepts, both across studies and within the same studies."

XXvi11 A ce propos, Meier suggère (1995:352): "Where then are universals to be sought? Because politeness as socially appropriate behavior must be viewed within a framework of social interaction, a search for universals must be found therein as well. Norms for appropriate behavior will certainly vary across cultures as will the underlying value and belief systems which, arising from an interplay of factors (e.g. political, social, historical), inform these norms. What all societies do have in common, however, is the existence of norms for appropriate behavior."

xxix On retrouve ici le positionnement de Goffman, à savoir, dans l'interaction face à face on observe l'influence réciproque que les participants exercent sur leurs actions respectives lorsqu'ils sont en présence physique immédiate les uns des autres.

XXX Comme l'écrit Olivier Maulini (2009:1) « «Dis bonjour à la dame ! » «h, tu dis 
pas bonjour?! » Nous avons tous entendu ces phrases une fois ou l'autre dans notre vie. »)

Xxxi On constate néanmoins que «si d'un côté les enfants se montrent parfois réticents à dire bonjour aux personnes qu'ils ne connaissent pas [...] [ils] aiment par contre que leurs camarades ne les ignore pas. » Maulini 2009: 1

XXX1i Dans Petites chroniques à mots couverts, Martine Boujou (2011) traite des lieux communs transmis de génération en génération (comme le rituel des salutations) en tant que forme d'autoritarisme et donc de violence faite aux enfants.

Xxxiii « Politesse » devint à la mode dans la France du XVIIe siècle pendant lequel Mme de Scudéry consacra un de ses dialogues au sujet, le définissant comme " savoir-vivre ». Sur ce sujet, Burke (2007).

xxxiv Article intégral : http://www.leparisien.fr/archives/c-est-combien-le-cafe-cadepend- de-votre-politesse-13-03-2016-621387.php Date de consulte : 01/12/2019

XXXV Depuis, la version originale a été reprise et reformulée par bien d'autres commerçant.e.s.

xxxvi Selon Le Parisien du 13 mars 2016, alors qu'au 03 mars 2016, le Huffington Post fait déjà état d'environ 3500 internautes.https://www.huffingtonpost.fr/2016/03/03/prixcafe-politesse-restaurant-francais_n 9372238.html

XXXVil Reddit https://www.reddit.com/r/pics/comments/481vnl/un_caf\%C3\%A9/

xxxviii Date de consulte : 20/11/2019. http://www.leparisien.fr/archives/c-est-combienle- cafe-ca-depend-de-votre-politesse-13-03-2016-5621387.php

XXxix Date de consulte 1/12/2019: http://www.leparisien.fr/archives/c-est-combien-lecafe- ca-depend-de-votre-politesse-13-03-2016-5621387.php

X1 Date de consulte 1/12/2019:

https://www.reddit.com/r/learnfrench/comments/48mtjw/un_café xpost_from_rpics/ ${ }_{\text {Xl1 }}$ Date de consulte 1/12/2019: https://www.reddit.com/r/pics/comments/48lvnl/un_café/

xlii Date de consulte 1/12/2019:

xliii Date de consulte 10/11/2019:

https://www.reddit.com/r/pics/comments/481vnl/un_café/

xliv Date de consulte 1/12/2019 :

https://www.reddit.com/r/learnfrench/comments/48mtjw/un_café_xpost_from_rpics/ xlv Date de consulte 1/12/2019 :

https://www.reddit.com/r/learnfrench/comments/48mtjw/un_café_xpost_from_rpics/

${ }^{\mathrm{Xlv1}}$ Date de consulte 1/12/2019 :

https://www.reddit.com/r/pics/comments/481vnl/un_café/ 